Rev. Bras. Saúde Prod. Anim., Salvador, v.16, n.1, p.179-189jan./mar., 2015 http://www.rbspa.ufba.br ISSN 15199940

\title{
Uso preventivo do toltrazuril para controle da coccidiose em cabritos de corte criados em região semiárida
}

\author{
Preventive use of toltrazuril to control coccidiosis in meat goats raised in semiarid \\ region
}
NUNES, Deisiane Moreira ${ }^{1 *}$; CRUZ, Jurandir Ferreira ${ }^{2}$; TEIXEIRA NETO, Milton Rezende $^{3}$

\author{
${ }^{1}$ Universidade Estadual do Sudoeste da Bahia, Programa de Pós-Graduação em Zootecnia, Itapetinga, \\ Bahia, Brasil. \\ ${ }^{2}$ Universidade Estadual do Sudoeste da Bahia, Departamento de Fitotecnia e Zootecnia, Vitória da \\ Conquista, Bahia, Brasil. \\ ${ }^{3}$ Faculdade de Tecnologia e Ciências, Vitória da Conquista, Bahia, Brasil. \\ *Endereço para correspondência: deisiane.ms@ @otmail.com
}

\section{RESUMO}

Objetivou-se com este estudo avaliar a eficácia do toltrazuril no controle da coccidiose em cabritos de corte criados extensivamente em região semiárida e determinar a idade mais adequada para realização do tratamento. Quarenta cabritos foram separados por faixa etária e tratados com toltrazuril em dose única aos 14; 21; 28 e 35 dias de idade. Amostras de fezes foram coletadas semanalmente para determinação do número de oocistos por grama de fezes (Oopg) nas semanas subsequentes ao tratamento. Os animais também foram pesados semanalmente para determinação do ganho de peso médio diário (GPD). Todos os cabritos tratados não apresentaram oocistos nas fezes pelo menos por uma semana após o tratamento. O Oopg dos cabritos tratados, independentemente da idade do tratamento, foi menor do que os não tratados $(\mathrm{P}<0,05)$ ao longo de 10 -11 semanas pós-tratamento. $\mathrm{O}$ ganho de peso médio diário (GPD) foi similar em todos os tratamentos avaliados. O Oopg não foi influenciado pelo sexo, mas sim pela idade ao tratamento. Os animais tratados aos 21 dias de idade não apresentaram oocistos nas fezes por um período maior que os demais. $\mathrm{O}$ tratamento com toltrazuril realizado aos 21 dias de idade foi eficaz para controle da coccidiose em cabritos criados extensivamente no semiárido.

Palavras-chave: anticoccidiano, cabritos, ganho de peso diário, idade ao tratamento

\section{SUMMARY}

This study aims to evaluate the effectiveness of toltrazuril in controlling coccidiosis in meat goats raised extensively in semiarid region and determine the most appropriate age for performing treatment. Forty kids were divided by age and treated with single dose of toltrazuril at 14, 21, 28 and 35 days aged. Fecal samples were collected weekly to determine the number of oocysts per gram of feces (Oopg) after treatment. The kids were weighed weekly to determine average daily gain (ADG). All treated kids showed no oocysts in the feces by at least one week after treatment. The Oopg of treated kids regardless of age at treatment was lower than the untreated $(\mathrm{P}<0.05)$ along $10-11$ weeks post-treatment. The ADG was similar for all treatments evaluated. The Oopg was not influenced by sex but it was by age at treatment. Kids treated at 21 days aged showed no oocysts in the feces for a longer period than the other. Preventive treatment with toltrazuril performed at 21 days aged was suitable for control of coccidiosis in kids raised extensively in semiarid region.

Keywords: anticocidial, goat kids, average daily gain, age at treatment 
Rev. Bras. Saúde Prod. Anim., Salvador, v.16, n.1, p.179-189jan./mar., 2015 http://www.rbspa.ufba.br ISSN 15199940

\section{INTRODUÇÃO}

A coccidiose é uma infecção parasitária, causada por protozoários do gênero Eimeria, que se constitui em sério problema para a caprinocultura devido às várias alterações orgânicas ocasionadas ao hospedeiro (FREITAS et al., 2005).

Em programas de prevenção da coccidiose é essencial o estabelecimento de manejo integrado que inclua o uso de agentes anticoccidianos (IQBAL et al., 2013), uma vez que o controle dessa enfermidade depende da redução da contaminação ambiental por oocistos infectantes (FRANDSEN et al. 1992).

Devido ao fato dos animais jovens apresentarem imunidade reduzida diante da infecção pelos coccídeos (BONFIM \& LOPES, 1994), o impacto econômico decorrente da elevada morbidade e mortalidade causada pela coccidiose, ocorre principalmente nesta categoria animal (LIMA, 2004; SILVA, 2007). No entanto, a despeito dos prejuízos advindos das infecções coccidianas, este problema ainda permanece sem solução efetiva (ANDREWS, 2013).

$\mathrm{O}$ tratamento preventivo de todo $\mathrm{o}$ rebanho susceptível, especialmente dos animais jovens, iniciado imediatamente após o contato com as formas infectantes é mais eficaz do que o tratamento curativo (VIEIRA et al., 2004). Por outro lado, os compostos quimioterápicos utilizados devem ser isentos de efeitos colaterais indesejáveis e agirem de forma rápida na redução da quantidade de oocistos fecais e na cura dos animais jovens (FRANDSEN et al. 1992).

O toltrazuril, derivado da triazina, é um agente quimioterápico comumente utilizado na avicultura e suinocultura para o tratamento da coccidose, que não apresenta nenhuma relação química com outros anticoccidianos utilizados na espécie caprina. Esse fato sugere a inexistência de resistência das espécies de Eimeria que parasitam os caprinos (FRANDSEN et al., 1992). Assim, objetivou-se com este estudo avaliar a eficácia do toltrazuril no controle da coccidiose em cabritos de corte criados em região semiárida.

\section{MATERIAL E MÉTODOS}

O estudo foi realizado em uma propriedade rural do município de Brumado - BA $\left(13^{\circ} 39^{\prime} 13^{\prime \prime} \mathrm{S}\right.$, $41^{\circ} 50$ '37'O), a $422 \mathrm{~m}$ de altitude, localizado na região Sudoeste da Bahia, situado na Depressão Sertaneja Meridional do bioma caatinga (VELLOSO et al., 2002). O clima da região é classificado como semiárido Bsh, segundo a classificação KöeppenGeiger (PEEL et al., 2007), apresentando temperatura anual média de $27^{\circ} \mathrm{C}$ e precipitação pluviométrica anual de 582 $\mathrm{mm}$, no período de janeiro a dezembro de 2013 (INMET, 2014), conforme apresentado na Figura 1.

$\mathrm{O}$ ensaio foi conduzido durante os meses de abril a julho de 2013, totalizando 77 dias de avaliação. Foram utilizados 40 cabritos lactentes, F1(Bôer $x$ SRD), de ambos os sexos, criados em sistema extensivo, com escore corporal entre 2,5 e 3,0. Nas duas primeiras semanas de vida, os cabritos foram criados separados das respectivas mães e amamentados naturalmente duas vezes por dia (início da manhã e final da tarde). Nas semanas seguintes foram mantidos com as matrizes em pastagem nativa da caatinga, recebendo sal mineral (Caprinofós®, Tortuga, Brasil) e água ad libitum.

Os cabritos foram distribuídos em cinco grupos (G14; G21; G28; G35 e GC), com oito animais cada, classificados de acordo com a faixa etária, ou seja, 14, $21,28,35$ e 28 dias de idade. Todos os 
Rev. Bras. Saúde Prod. Anim., Salvador, v.16, n.1, p.179-189jan./mar., 2015 http://www.rbspa.ufba.br ISSN 15199940

cabritos, exceto aqueles do grupo controle (GC), foram tratados com toltrazuril (Baycox ® 5\%, Bayer, Brasil) na dose $20 \mathrm{mg} / \mathrm{kg}$ p.v., administrado por via oral, uma única vez na ocasião da divisão dos grupos. Aos cabritos do GC foi administrado somente água, na dose de $1,0 \mathrm{~mL} / 3,5 \mathrm{~kg}$ p.v. aos 28 dias de idade (Tabela 1).

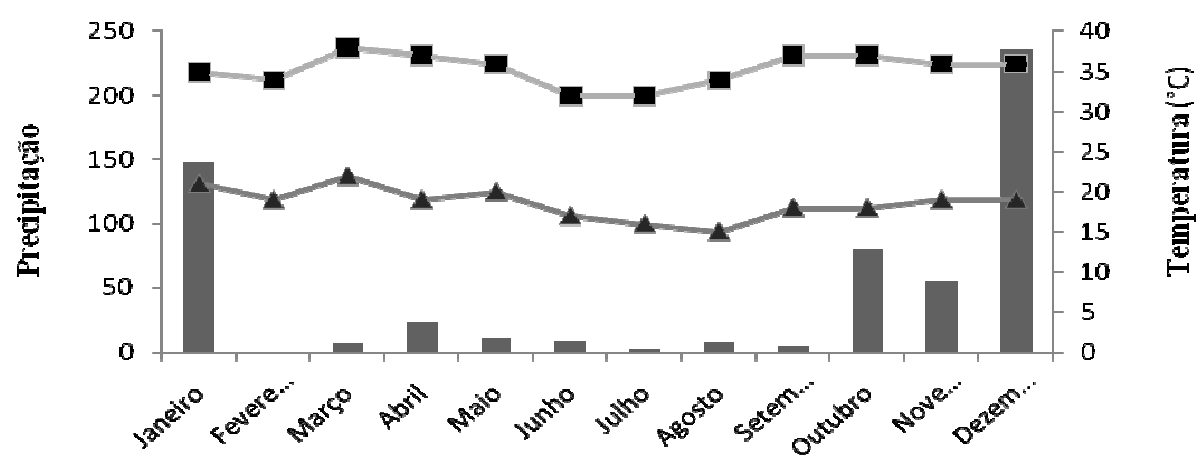

Figura 1. Temperaturas mínima e máxima $(-\boldsymbol{\Lambda} ;-)$ e precipitação pluviométrica ( ( ) no período de janeiro a dezembro de 2013 (INMET, 2014)

Tabela 1. Grupos de cabritos submetidos ou não ao tratamento com toltrazuril em diferentes idades

\begin{tabular}{ccccc}
\hline Grupo & Idade & $\mathrm{N}^{\circ}$ de animais & Tratamento & Dose \\
\hline G14 & 14 dias & 08 & $*$ toltrazuril & $20 \mathrm{mg} / \mathrm{kg} \mathrm{p.v}$. \\
G21 & 21 dias & 08 & $*$ toltrazuril & $20 \mathrm{mg} / \mathrm{kg} \mathrm{p.v}$. \\
G28 & 28 dias & 08 & *toltrazuril & $20 \mathrm{mg} / \mathrm{kg} \mathrm{p.v}$. \\
G35 & 35 dias & 08 & $*$ toltrazuril & $20 \mathrm{mg} / \mathrm{kg} \mathrm{p.v.}$ \\
GC & 28 dias & 08 & água & $1,0 \mathrm{~mL} / 3,5 \mathrm{~kg} \mathrm{p} . \mathrm{v}$ \\
\hline
\end{tabular}

**Baycox ${ }^{\circledR}$ 5\%, Bayer, Brasil; GC = grupo controle.

Semanalmente, cada animal foi pesado em balança digital para a determinação do peso vivo e do ganho de peso. Neste momento também foi observada a aparência geral dos animais. Após a pesagem, amostras de fezes foram coletadas diretamente da ampola retal, armazenadas em sacos plásticos individualmente identificados, acondicionados em recipiente isotérmico contendo gelo reciclável e enviadas imediatamente ao laboratório. As análises foram realizadas no Laboratório de Biologia do Instituto Federal de Educação, Ciência e Tecnologia Baiano Campus Itapetinga, até 48 horas após a coleta.
As amostras fecais foram processadas de acordo com a técnica McMaster de flutuação desenvolvida por Gordon \& Whitlock (1939) e modificada por Ueno \& Gonçalves (1998). Dois gramas de fezes de cada amostra foram maceradas e diluídas em $58 \mathrm{~mL}$ de solução saturada de cloreto de sódio e a suspensão resultante foi filtrada em peneira de malha fina; uma pequena alíquota foi coletada e utilizada para preencher as retículas da câmara de McMaster; a leitura foi procedida em microscópio óptico (40X) e a quantidade oocistos por grama de fezes foi determinada pelo número de oocistos de Eimeria sp encontrados x 
Rev. Bras. Saúde Prod. Anim., Salvador, v.16, n.1, p.179-189jan./mar., 2015 http://www.rbspa.ufba.br ISSN 15199940

100. De acordo com o número de oocistos encontrados, a intensidade de infecção foi classificada em baixa $\left(<1.10^{3}\right)$, moderada $\left(1.10^{3}-1.10^{4}\right)$ e alta $\left(>1.10^{4}\right), \quad($ UENO \& GONÇALVES, 1998).

$\mathrm{Na}$ análise dos resultados, os dados referentes ao Oopg (quantidade de oocistos por grama de fezes) foram previamente transformados em logaritmos. Os dados (médias \pm EP) de Oopg nas diferentes idades e o ganho de peso diário foram comparados pelo teste de Student-Newman-Keuls do PROC GLM, SAS versão 9.1 (STATISTICAL ANALYSIS SYSTEM, 2003). Os valores de Oopg de machos e fêmeas foram comparados pelo teste $\mathrm{t}$ de Student (EXCEL, 2007). As diferenças foram consideradas significativas com $\mathrm{P}<0,05$.

\section{RESULTADOS E DISCUSSÃO}

Em $100 \%$ das amostras fecais analisadas no início do estudo foram encontrados oocistos de Eimeria sp, revelando a marcante presença dessa enfermidade entre os animais jovens (Tabela 2).

As infecções frequentemente ocorrem durante os primeiros dias após o nascimento e pode rapidamente conduzir a eliminação de oocistos, dependendo da duração média do período pré-patente das espécies de Eimeria envolvidas (RUIZ et al., 2014). $\mathrm{O}$ aparecimento de oocistos nas fezes dos cabritos com apenas 14 dias de idade indicou que a infecção ocorreu nos primeiros dias de vida, sugerindo infecção por espécies de Eimeria com período pré-patente curto (LIMA, 1981; DAI et al., 2006; RAKHSHANDEHROO et al., 2013; RUIZ et al., 2013).

$\mathrm{O}$ uso preventivo do toltrazuril no controle da coccidiose tem sido recomendado a partir da $3^{\mathrm{a}}$ semana de vida ou 10-14 dias após exposição ao ambiente contaminado ou ao primeiro pastejo em bezerros (EPE et al., 2005) e cordeiros (DIAFERIA et al., 2013). Em cabritos, Iqbal et al. (2013) sugeriram o uso do toltrazuril no primeiro dia de exposição dos animais ao ambiente contaminado.

No presente estudo, a administração do toltrazuril aos 14 dias de idade manteve a intensidade de infecção em nível similar nos grupos tratados aos 21, $28 \mathrm{e}$ 35 dias de idade $(\mathrm{P}>0,05)$ durante todo o período monitorado (10 semanas póstratamento). Esses resultados refutam a afirmativa de Gjerde \& Helle (1991), os quais sugerem que o tratamento realizado em animais muito jovens elimina os coccídios antes que o sistema imune do hospedeiro tenha sido adequadamente estimulado pela infecção, deixando o animal parcialmente susceptível à coccidiose após o término do efeito do fármaco.

A quantidade de oocistos liberados nas fezes dos animais tratados com toltrazuril permaneceu menor $(\mathrm{P}<0,05)$ durante 10 11 semanas subsequentes ao tratamento em relação ao grupo controle (GC), independentemente da idade em que ocorreu a administração do fármaco. Esses resultados reforçam a afirmativa de Steinfelder et al. (2005) que o tratamento com toltrazuril durante a primo-infecção não afeta o desenvolvimento da resposta celular de defesa, permitindo o estabelecimento da imunidade específica contra os parasitos e prevenindo a ocorrência da coccidiose clínica.

Com exceção dos cabritos tratados aos 14 dias de idade (G14), os quais apresentaram valores de Oopg inferiores aos demais na $9^{\mathrm{a}}$ semana pós-tratamento $(\mathrm{P}<0,05)$, os animais dos grupos $\mathrm{G} 21$, G28 e G35 apresentaram oocistogramas similares no período entre a $7^{\mathrm{a}}$ e a $11^{\mathrm{a}}$ 
Rev. Bras. Saúde Prod. Anim., Salvador, v.16, n.1, p.179-189jan./mar., 2015 http://www.rbspa.ufba.br ISSN 15199940

semana pós-tratamento $(\mathrm{P}>0,05)$ (Tabela 2). Ressalta-se que, durante o período experimental, não foi verificada diferença clínica entre animais dos grupos tratados.

O toltrazuril age sobre todos os estágios de desenvolvimento intracelular da Eimeria sp (GJERDE \& HELLE, 1991), mas não afeta os estágios de vida extracelular (esporozoítos e merozoítos), permitindo-lhes que penetrem nas células epiteliais ou da lâmina própria da mucosa, e assim, induzam o desenvolvimento da imunidade natural (DENIZ, 2008). Além disso, os estágios de Eimeria sp. danificados pela ação do toltrazuril, permanecem nas células do hospedeiro agindo como antígenos e portanto estimulando o sistema imunológico (GREIF, 2000).

Tabela 2. Quantidade de oocistos por grama de fezes (Oopg) em diferentes momentos pré e pós-tratamento com toltrazuril em cabritos de corte criados em região semiárida

\begin{tabular}{cccccc}
\hline $\begin{array}{c}\text { Semanas } \\
\text { de pós - } \\
\text { tratamento }\end{array}$ & $\mathrm{G} 14$ & $\mathrm{G} 21$ & $\mathrm{G} 28$ & $\mathrm{G} 35$ & $\mathrm{GC}$ \\
\cline { 2 - 6 } & $59.900 \pm 3.049^{\mathrm{A}}$ & $93.066 \pm 16.039^{\mathrm{A}}$ & $63.600 \pm 16.039^{\mathrm{A}}$ & $87.360 \pm 2.564^{\mathrm{A}}$ & $66.214 \pm 23.383^{\mathrm{A}}$ \\
1 & $0^{\mathrm{B}}$ & $0^{\mathrm{B}}$ & $0^{\mathrm{B}}$ & $0^{\mathrm{B}}$ & $34.887 \pm 9.192^{\mathrm{A}}$ \\
2 & $12,5 \pm 12,5^{\mathrm{B}}$ & $0^{\mathrm{B}}$ & $0^{\mathrm{B}}$ & $0^{\mathrm{B}}$ & $29.212 \pm 8.594^{\mathrm{A}}$ \\
3 & $37,5 \pm 18,3^{\mathrm{BC}}$ & $0^{\mathrm{C}}$ & $12,5 \pm 12,5^{\mathrm{BC}}$ & $862,5 \pm 819^{\mathrm{B}}$ & $29.837 \pm 8.768^{\mathrm{A}}$ \\
4 & $525 \pm 193^{\mathrm{B}}$ & $12,5 \pm 12,5^{\mathrm{C}}$ & $75 \pm 36,5^{\mathrm{BC}}$ & $1.175 \pm 846^{\mathrm{B}}$ & $35.412 \pm 10.794^{\mathrm{A}}$ \\
5 & $937 \pm 303^{\mathrm{BC}}$ & $387,5 \pm 137^{\mathrm{C}}$ & $712,5 \pm 174^{\mathrm{BC}}$ & $2.950 \pm 1.435^{\mathrm{B}}$ & $42.987 \pm 9.916^{\mathrm{A}}$ \\
6 & $2.775 \pm 1.723^{\mathrm{B}}$ & $812 \pm 240^{\mathrm{C}}$ & $5.071 \pm 2.036^{\mathrm{B}}$ & $7.375 \pm 4.219^{\mathrm{B}}$ & $40.828 \pm 11.499^{\mathrm{A}}$ \\
7 & $5.383 \pm 2.330^{\mathrm{B}}$ & $1.285 \pm 278^{\mathrm{B}}$ & $9.112 \pm 4.060^{\mathrm{B}}$ & $12.525 \pm 6.537^{\mathrm{B}}$ & $45.237 \pm 10.810^{\mathrm{A}}$ \\
8 & $7.775 \pm 2.094^{\mathrm{B}}$ & $6.466 \pm 1.735^{\mathrm{B}}$ & $21.328 \pm 7.540^{\mathrm{B}}$ & $19.187 \pm 6.510^{\mathrm{B}}$ & $87.700 \pm 30.498^{\mathrm{A}}$ \\
9 & $13.216 \pm 2.464^{\mathrm{C}}$ & $17.241 \pm 1.560^{\mathrm{BC}}$ & $30.116 \pm 3.664^{\mathrm{B}}$ & $29.043 \pm 7.327^{\mathrm{B}}$ & $99.200 \pm 28.074^{\mathrm{A}}$ \\
10 & $23.233 \pm 4.211^{\mathrm{B}}$ & $28.033 \pm 2.473^{\mathrm{B}}$ & $31.225 \pm 4.495^{\mathrm{B}}$ & $35.657 \pm 5.091^{\mathrm{B}}$ & $133.475 \pm 47.097^{\mathrm{A}}$ \\
11 & - & $34.925 \pm 4.423^{\mathrm{B}}$ & $34.657 \pm 7.093^{\mathrm{B}}$ & $50.617 \pm 4.793^{\mathrm{B}}$ & $181.500 \pm 62.158^{\mathrm{A}}$ \\
\hline $\mathrm{A}, \mathrm{B}, \mathrm{C}$ & Valores com letras diferentes, na mesma linha, diferem pelo teste Student-Newman-Keuls $(\mathrm{P}<0,05)$.
\end{tabular}

O controle advindo do tratamento com o toltrazuril interferiu na intensidade da infecção entre a $8^{\mathrm{a}}$ e $10^{\mathrm{a}}$ semana de idade, sendo menor nos animais tratados aos 21 e 35 dias de vida $(\mathrm{P}<0,05)$. Os animais tratados aos 14 dias de idade apresentaram alta intensidade de infecção (Oopg > 1.10 ${ }^{4}$ ) uma semana antes dos demais grupos tratados. Os animais não tratados apresentaram alta intensidade de infecção em todas as idades avaliadas (Tabela 3).

Tabela 3. Quantidade de oocistos por grama de fezes (Oopg) eliminados entre $8^{\mathrm{a}}$ e $12^{\mathrm{a}}$ semana de idade em cabritos de corte tratados ou não com toltrazuril

\begin{tabular}{cccccc}
\hline Idade & \multicolumn{5}{c}{ Oopg (média $\pm \mathrm{EP})$} \\
\cline { 2 - 6 } (semanas) & $\mathrm{G} 14$ & $\mathrm{G} 21$ & $\mathrm{G} 28$ & $\mathrm{G} 35$ & $\mathrm{GC}$ \\
\hline 8 & $2.775 \pm 1.723^{\mathrm{B}}$ & $387,5 \pm 137^{\mathrm{B}}$ & $75 \pm 36,5^{\mathrm{B}}$ & $862,5 \pm 819^{\mathrm{B}}$ & $35.412 \pm 10.794^{\mathrm{A}}$ \\
9 & $5.383 \pm 2.330^{\mathrm{B}}$ & $812 \pm 240^{\mathrm{B}}$ & $712,5 \pm 174^{\mathrm{B}}$ & $1.175 \pm 846^{\mathrm{B}}$ & $42.987 \pm 9.916^{\mathrm{A}}$ \\
10 & $7.775 \pm 2.094^{\mathrm{B}}$ & $1.285 \pm 278^{\mathrm{D}}$ & $5.071 \pm 2.036^{\mathrm{BC}}$ & $2.950 \pm 1.435^{\mathrm{CD}}$ & $40.828 \pm 11.499^{\mathrm{A}}$ \\
11 & $13.216 \pm 2.464^{\mathrm{AB}}$ & $6.466 \pm 1.735^{\mathrm{B}}$ & $9.112 \pm 4.060^{\mathrm{B}}$ & $7.375 \pm 4.219^{\mathrm{B}}$ & $45.237 \pm 10.810^{\mathrm{A}}$ \\
12 & $23.233 \pm 4.211^{\mathrm{B}}$ & $17.241 \pm 1.560^{\mathrm{B}}$ & $21.328 \pm 7.540^{\mathrm{B}}$ & $12.525 \pm 6.537^{\mathrm{B}}$ & $87.700 \pm 30.498^{\mathrm{A}}$ \\
\hline $\mathrm{A}, \mathrm{B}, \mathrm{C}, \mathrm{D}$ & Valores com letras diferentes, na mesma linha, diferem pelo teste Student-Newman-Keuls $(\mathrm{P}<0,05)$.
\end{tabular}


Rev. Bras. Saúde Prod. Anim., Salvador, v.16, n.1, p.179-189jan./mar., 2015 http://www.rbspa.ufba.br ISSN 15199940

A aparente variação na eficácia do fármaco em função da idade dos animais pode ser devida às diferentes fases de desenvolvimento dos coccídios no momento do tratamento, uma vez que a ação do toltrazuril é limitada aos estágios intracelulares do parasito (GREIF, 2000). A proporção de estágios de Eimeria sp. susceptíveis ao toltrazuril, provavelmente diferem em função da formação de merontes $\mathrm{e}$ gamontes e a duração da replicação nas células do hospedeiro (DENIZ, 2008), sugerindo que o momento de realização do tratamento preventivo interfere na dinâmica de desenvolvimento e eliminação dos oocistos.

No grupo de animais sem tratamento anticoccidiano (GC), foi possível observar uma expressiva redução na contagem de Oopg, a partir dos 35 dias de idade, sugerindo a ocorrência de um pico de eliminação de oocistos quando os animais estavam com $\leq 28$ dias de vida (Figura 2). O Oopg se manteve estável até os 70 dias de idade, provavelmente devido ao desenvolvimento de imunidade parcial na primo-infecção (DAI et al., 2006). Por outro lado, a partir dos 77 dias de idade houve elevação progressiva na contagem de Oopg, alcançando valor superior a $1,8.10^{5}$ ao final do experimento (Figura 2).

Esses resultados diferem de estudos prévios que sugerem a ocorrência de um segundo pico de eliminação de oocistos, seguido de expressiva redução, aos 56 dias de vida (FRANDSEN et al.,1992; VIEIRA et al., 2004; LIMA et al., 2009; SILVA et al., 2007; RAKHSHANDEHROO et al., 2013). Por outro lado, a não redução do Oopg após a $8^{a}$ semana de vida sugere que a imunidade adquirida na primo-infecção pode ter sido limitada a despeito do alto grau de exposição ao parasito (CHAPMAN et al., 1973; DAI et al., 2006).
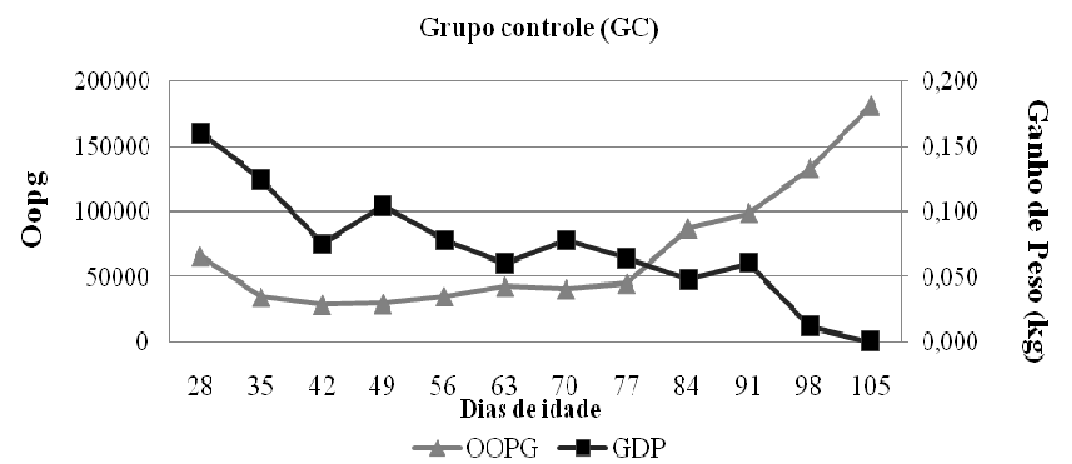

Figura 2. Quantidade de oocistos por grama de fezes (Oopg) e ganho de peso diário (GPD) em cabritos de corte dos 28 aos 105 dias de idade, sem tratamento anticocidiano e criados em região semiárida

De acordo com Ruiz et al. (2013), cabritos com 70 a 84 dias de idade, são particularmente sensíveis a coccidiose clínica, podendo refletir numa falta de imunidade protetora nessa idade, mesmo tendo tolerado infecções anteriores com espécies patogênicas de Eimeria sp. A imunidade protetora desenvolvida contra Eimeria sp. não previne infecções subsequentes e manifestação dos sinais clínicos da doença, quando o hospedeiro apresenta condição imunológica comprometida por deficiências 
Rev. Bras. Saúde Prod. Anim., Salvador, v.16, n.1, p.179-189jan./mar., 2015 http://www.rbspa.ufba.br ISSN 15199940

nutricionais, estresse e/ou doenças concomitantes (LUCAS et al., 2006).

Tem sido relatada elevada intensidade de eliminação de oocistos $\left(>1.10^{4}\right.$ Oopg $)$ em cabritos com idade entre 2 a 4 meses, seguido por rápida diminuição do Oopg com o avanço da idade (CHARTIER et al., 1992; AGYEI et al., 2004; RUIZ et al., 2006). No presente estudo não foi possivel identificar a esperada redução na intensidade de eliminação de oocistos, visto que os animais apresentavam menos quatro meses de idade ao final do estudo.
A quantidade de oocistos eliminados não foi influenciada pelo sexo, independentemente da idade em que ocorreu o tratamento preventivo (Tabela 4). Esses resultados diferem de estudos prévios realizados com caprinos adultos, nos quais a despeito das fêmeas apresentarem maior prevalência de infecção por Eimeria sp, (REHMAN et al., 2011; SHARMA et al., 2009), os machos apresentaram maior intensidade de infecção (BRITO et al., 2009; FEITOSA et al., 2009).

Tabela 4. Quantidade de oocistos de Eimeria sp. eliminados por cabritos machos e fêmeas criados em região semiárida, tratados ou não com toltrazuril em diferentes idades

\begin{tabular}{ccc}
\hline \multirow{2}{*}{ Idade ao tratamento (dias) } & \multicolumn{2}{c}{ Oopg (média \pm EP) } \\
\cline { 2 - 3 } & Machos & Fêmeas \\
\hline 14 & $9.913 \pm 3.017^{\mathrm{A}}$ & $10.235 \pm 2.785^{\mathrm{A}}$ \\
21 & $33.054 \pm 23.953^{\mathrm{A}}$ & $10.038 \pm 2.578^{\mathrm{A}}$ \\
28 & $11.287 \pm 2.295^{\mathrm{A}}$ & $17.639 \pm 4.596^{\mathrm{A}}$ \\
GC & $17.894 \pm 4.361^{\mathrm{A}}$ & $18.618 \pm 3.571^{\mathrm{A}}$ \\
\hline
\end{tabular}

Valores com letras iguais, na mesma linha, não diferem pelo Teste t de Student $(\mathrm{P}<0,05)$.

$\mathrm{GC}=$ grupo sem tratamento.

No que ser refere ao desempenho dos animais, o ganho de peso diário (GPD) apresentou perfil relativamente similar com valores variando entre 50 e 100 g/dia na maior parte do período póstratamento observado. $\mathrm{Na} 8^{\mathrm{a}}$ semana pós-tratamento houve redução do ganho de peso diário em todos os grupos tratados (Figura 3).

A redução no desempenho de todos os animais no mesmo momento póstratamento pode ter sido consequência do sistema de criação e fonte de alimentação dos cabritos, visto que os mesmos eram lactantes. As matrizes (SRD) cuja base alimentar é a vegetação da Caatinga, podem apresentar redução drástica na produção de leite após o pico de lactação que ocorre aos 45 dias pós- parto (RIBEIRO, 1998).

Apesar da expressiva redução do Oopg nos animais tratados preventivamente com toltrazuril, não houve diferença no desempenho dos animais tratados e não tratados (Tabela 5). Esse fato foi também observado em estudos prévios (CHARTIER et al., 1992; RUIZ et al., 2014) realizados com cabritos tratados contra a coccidiose.

A não observância de diferença entre animais tratados e não tratados pode ser consequência da metodologia comumente utilizada nos estudos. Em ambientes altamente contaminados com oocistos de Eimeria sp. o risco de desenvolvimento da doença é geralmente elevado (RUIZ et al., 2014). 
Rev. Bras. Saúde Prod. Anim., Salvador, v.16, n.1, p.179-189jan./mar., 2015 http://www.rbspa.ufba.br
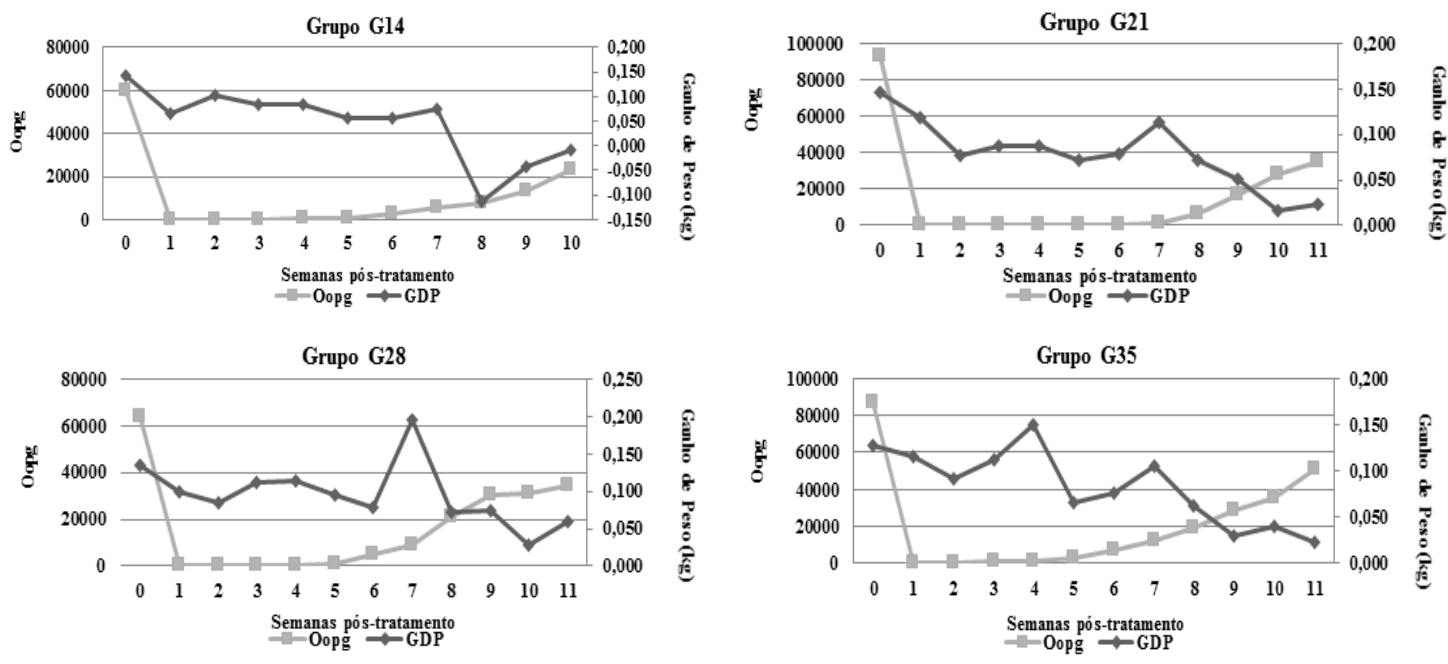

Figura 3. Quantidade de oocistos por grama de fezes (Oopg) e ganho de peso diário (GPD) antes e após administração do toltrazuril aos 14, 21, 28 e 35 dias de idade em cabritos de corte criados em região semiárida

Tabela 5. Ganho de peso diário de cabritos de corte criados em região semiárida, tratados ou não com toltrazuril em diferentes idades

\begin{tabular}{cccccc}
\hline $\begin{array}{c}\text { Semanas de } \\
\text { pós }- \\
\text { tratamento }\end{array}$ & G14 & G21 & G28 & G35 & GC \\
\cline { 2 - 6 } & $0,144 \pm 0,018^{\mathrm{A}}$ & $0,146 \pm 0,017^{\mathrm{A}}$ & $0,136 \pm 0,020^{\mathrm{A}}$ & $0,127 \pm 0,013^{\mathrm{A}}$ & $0,161 \pm 0,009^{\mathrm{A}}$ \\
1 & $0,066 \pm 0,036^{\mathrm{A}}$ & $0,118 \pm 0,020^{\mathrm{A}}$ & $0,098 \pm 0,008^{\mathrm{A}}$ & $0,116 \pm 0,017^{\mathrm{A}}$ & $0,125 \pm 0,010^{\mathrm{A}}$ \\
2 & $0,104 \pm 0,021^{\mathrm{A}}$ & $0,077 \pm 0,024^{\mathrm{A}}$ & $0,084 \pm 0,019^{\mathrm{A}}$ & $0,091 \pm 0,010^{\mathrm{A}}$ & $0,075 \pm 0,012^{\mathrm{A}}$ \\
3 & $0,084 \pm 0,021^{\mathrm{A}}$ & $0,088 \pm 0,020^{\mathrm{A}}$ & $0,112 \pm 0,014^{\mathrm{A}}$ & $0,112 \pm 0,018^{\mathrm{A}}$ & $0,105 \pm 0,015^{\mathrm{A}}$ \\
4 & $0,084 \pm 0,014^{\mathrm{A}}$ & $0,087 \pm 0,026^{\mathrm{A}}$ & $0,114 \pm 0,017^{\mathrm{A}}$ & $0,150 \pm 0,025^{\mathrm{A}}$ & $0,078 \pm 0,004^{\mathrm{A}}$ \\
5 & $0,057 \pm 0,012^{\mathrm{A}}$ & $0,071 \pm 0,020^{\mathrm{A}}$ & $0,095 \pm 0,011^{\mathrm{A}}$ & $0,066 \pm 0,026^{\mathrm{A}}$ & $0,060 \pm 0,004^{\mathrm{A}}$ \\
6 & $0,055 \pm 0,010^{\mathrm{A}}$ & $0,078 \pm 0,013^{\mathrm{A}}$ & $0,078 \pm 0,009^{\mathrm{A}}$ & $0,076 \pm 0,013^{\mathrm{A}}$ & $0,078 \pm 0,005^{\mathrm{A}}$ \\
7 & $0,076 \pm 0,020^{\mathrm{A}}$ & $0,114 \pm 0,019^{\mathrm{A}}$ & $0,195 \pm 0,014^{\mathrm{A}}$ & $0,105 \pm 0,010^{\mathrm{A}}$ & $0,064 \pm 0,005^{\mathrm{A}}$ \\
8 & $-0,112 \pm 0,112^{\mathrm{A}}$ & $0,071 \pm 0,015^{\mathrm{A}}$ & $0,073 \pm 0,016^{\mathrm{A}}$ & $0,063 \pm 0,015^{\mathrm{A}}$ & $0,048 \pm 0,008^{\mathrm{A}}$ \\
9 & $-0,041 \pm 0,011^{\mathrm{B}}$ & $0,051 \pm 0,016^{\mathrm{A}}$ & $0,073 \pm 0,006^{\mathrm{A}}$ & $0,030 \pm 0,016^{\mathrm{A}}$ & $0,061 \pm 0,006^{\mathrm{A}}$ \\
10 & $-0,01 \pm 0,020^{\mathrm{B}}$ & $0,016 \pm 0,018^{\mathrm{AB}}$ & $0,029 \pm 0,017^{\mathrm{AB}}$ & $0,039 \pm 0,013^{\mathrm{A}}$ & $0,013 \pm 0,013^{\mathrm{AB}}$ \\
11 & - & $0,023 \pm 0,018^{\mathrm{AB}}$ & $0,059 \pm 0,019^{\mathrm{A}}$ & $0,023 \pm 0,010^{\mathrm{AB}}$ & $0,00 \pm 0,009^{\mathrm{B}}$ \\
\hline
\end{tabular}

$* \mathrm{GPD}=$ ganho de peso médio diário; $\mathrm{GC}=$ grupo sem tratamento.

${ }^{\mathrm{A}, \mathrm{B}}$ Valores com letras diferentes, na mesma linha, diferem pelo teste Student-Newman-Keuls $(\mathrm{P}<0,05)$.

No entanto, a redução da contaminação ambiental promovida pelo tratamento da maioria dos animais contemporâneos avaliados no presente estudo, contribuiu para minimizar a exposição aos patógenos e, provavelmente, reduzir o impacto negativo da doença sobre os animais não tratados.

$\mathrm{O}$ uso do toltrazuril reduziu a intensidade de infecção por oocistos de Eimeria sp. em cabritos, e consequentemente, diminuiu a contaminação ambiental. O tratamento realizado aos 21 dias de idade foi mais eficaz para controle da coccidiose em cabritos criados extensivamente no semiárido em comparação com as outras idades. 
Rev. Bras. Saúde Prod. Anim., Salvador, v.16, n.1, p.179-189jan./mar., 2015 http://www.rbspa.ufba.br ISSN 15199940

\section{REFERÊNCIAS}

AGYEI, A.D.; ODONKOR, M.; OSEISOMUAH, A., Concurrence of Eimeria and helminth parasitic infections in West African Dwarf kids in Ghana. Small Ruminant Research, v.51, p.29-35, 2004.

ANDREWS, A.H. Some aspects of coccidiosis in sheep and goats. Small Ruminant Research, v.110, p.93-95, 2013.

BONFIM, T.C.B.; LOPES, C.W.G.

Levantamento de parasitos gastrintestinais em caprinos da Região Serrana do estado do Rio de Janeiro. Revista Brasileira de Parasitologia Veterinária, v.3; p.119-124, 1994.

BRITO, D.R.B.; SANTOS, A.C.G.; TEIXEIRA, W.C.; GUERRA, R.M. S.N.C. Parasitos gastrintestinais em caprinos e ovinos da microrregião do Alto do Mearim e Grajaú, no Estado do Maranhão, Brasil. Ciência Animal Brasileira, v.10, n.3, p.967-974, 2009.

CHAPMAN, H.D.; LEWIS, J.A.; SEARLE, R.M. The effect of naturally acquired infections of coccidian in lambs. Research in Veterinary Science, v.14, n.1, p.369 - 375, 1973.

CHARTIER, C.; PELLET, M.P.; PORS, I. Effects of toltrazuril on oocysts discharge and growth in kids with naturally-acquired coccidial infections.

Small Ruminant Research, v.8, p.171177, 1992.

DAI, Y.B.; LIU, X.Y.; LIU, M; TAO, J.P. Pathogenic effects of the cocciduim Eimeria ninakohlyakimovae in goats. Veterinary Research Communications, v.30, n.2, p.149-160, 2006.
DENIZ, A. Baycox® 5\% Toltrazuril coccidiocide for lamb. Germany, 2008 (Technical Manual - Bayer Health Care, Animal Health).

DIAFERIA, M.; VERONESI, F.; MORGANTI,G.; NISOLI, L.; FIORETTI, D.P. Efficacy of toltrazuril $5 \%$ suspension (Baycox, Bayer) and diclazuril (Vecoxan, Jassen-Cilag) in the controlo f Eimeria spp. in Lambs. Parasitology Research, v.112, p.163 168, 2013.

EPE, C.; SAMSONHIMMELSTJERNA, G.; WIRTHERLE, N.; HEYDEN, V.; WELZ, C.; BEENING, J.; RADELOFF, I.; HELLMANN, K.; SHNIEDER, T.; KRIEGER, K. Efficacy of toltrazuril as a metaphylactic and therapeutic treatment of coccidiosis in first-year grazing calves. Parasitology Research, v.97, p.127-133, 2005. Suppl. 1.

FEITOSA, J.V.; COUTINHO, C.R.; COSTA, A.N.L.; ARAÚJO, A.A.; COSTA, M.R.G.F.; TEIXEIRA, E.W.L.; MESQUITA, F.L.T. Estimativa do número de oocistos de eimeria em ovinos e caprinos do cariri cearense.

Revista Brasileira de Higiene e Sanidade Animal, v.3, n.2, p.7-13, 2009.

FRANDSEN, J.C.; BLAGBURN, B.L.; ARTHER, R.G. Toltrazuril (Bay Vi 9142) as an anticoccidial in naturally infected goats. Preventive Veterinary Medicine, v.12, p.101-110, 1992.

FREITAS, F.L.C.; ALMEIDA, K.S.; NASCIMENTO, A.A.; MACHADO, C.R.; VESCHI, J.L.A.; MACHADO, R.Z. Espécies do gênero Eimeria Schneider, 1875 (Apicomplexa: Eimeriidae) em caprinos leiteiros mantidos em sistema intensivo na região de São José do Rio Preto, Estado 
Rev. Bras. Saúde Prod. Anim., Salvador, v.16, n.1, p.179-189jan./mar., 2015 http://www.rbspa.ufba.br ISSN 15199940

de São Paulo, Brasil. Revista

Brasileira de Parasitologia

Veterinária, v.14, n.1, p.7-10, 2005.

GJERDE, B.; HELLE, O.

Chemoprophylaxis of coccidiosis in lambs with a single oral dose of toltrazuril. Veterinary Parasitology, v.38, p.97-107, 1991.

GORDON, H M.; WHITLOCK, H.V.A. New technique for counting nematodes eggs in sheep faeces. Journal of the Council for Scientifc and Industrial Research, v.12, p.50-52, 1939.

GREIF, G. Immunity to coccidiosis after treatment with toltrazuril. Parasitology

Research, v.86, p.787-790, 2000.

INSTITUTO NACIONAL DE METEOROLOGIA - INMET. Estações e dados. Disponível em <www.inmet.gov.br>. Acesso em: 18 maio 2014.

IQBAL, A.; TARIQ, K. A.; WAZIR, V.S.; SINGH, R. Antiparasitic efficacy of Artemisia absinthium, toltrazuril and amprolium against intestinal coccidiosis in goats. Journal Parasitic Diseases, v.37, n.1, p.88-93, 2013.

LIMA, J.D. Coccidiose dos Ruminantes Domésticos. In: CONGRESSO BRASILEIRO DE PARASITOLOGIA VETERINÁRIA \& I SIMPÓSIO LATINO-AMERICANO DE RICKETISIOSES, 8., 2004, Ouro Preto, MG. Anais... Ouro Preto, MG, 2004.

LIMA, J.D. Life cycle of Eimeria christenseni Levine, Ivens \& Fritz, 1962 form the domestic goat, Capra hircus L. Journal of Protozoology, v.28, n.1, p.5964, 1981.
LIMA, V.X.M; AHID, S.M.M.; SIMPLICIO, A.A. Efeito de sal mineral enriquecido ou não com ionóforos sobre a frequência de eimeriídeos de fêmeas caprinas jovens. Revista em Agronegócios e Meio Ambiente, v.2, n.2, p.63-71, 2009.

LUCAS, A.S.; SWECKER, W.S.; LINDSAY, D.S.; SCAGLIA, G.; ELVINGER, F.C.; ZAJAC, A.M. The effect of weaning method on coccidial infections in beef calves. Veterinary Parasitology, v.145, p.228-233, 2006.

PEEL, M.C.; FINLAYSON, B.L.; MCMAHON, T.C. Updated world map of the Koppen-Geiger climate classification. Hydrology and Earth System Sciences, v.11, p.1633-1644, 2007.

RAKHSHANDEHROO, E.; NAZIFI, S.; RAZAVI, M.; GHANE, M.; ALAVI,L A.M. Caprine Coccidiosis: the effects of induced infection on certain blood parameters. Veterinarski Arhiv, v.83, n.6, p.623 -631, 2013.

REHMAN, T.; KHAN, M.N.; KHAN, I.A.; AHAMAD, M. Epidemiology and Economic Benefits of Treating Goat Coccidiosis. Pakistan Veterinary Journal, v.31, n.3, p.227-230, 2011.

RIBEIRO, S.D.A. Caprinocultura: criação racional de caprinos. São Paulo: Nobel, 1998. 320p.

RUIZ, A.; GONZÁLEZ, J.F.; RODRÍGUEZ,E.; MARTÍN, B.S.; HERNÁNDEZ, Y.I.; ALMEIDA, R.; MOLINA, J.M. Influence of climatic and management factors on Eimeria infections in goats from semi-arid zones. The Journal Veterinary Medical Science, v.53, p.399-402, 2006. 
Rev. Bras. Saúde Prod. Anim., Salvador, v.16, n.1, p.179-189jan./mar., 2015 http://www.rbspa.ufba.br

RUIZ, A.; MUÑOZ, M.C.; MOLINA, J.M.; HERMOSILLA, C.; ANDRADA, M.; LARA, P.; BORDÓN, E.; PÉREZ, D.; LOPÉZ, A.M.; MATOS, L.; GUEDES, A.C.; FALCÓN, S.; FALCÓN, Y.; MARTÍN, S.; TAUBERT, A. Immunization with Eimeria ninakohlyakimovae -live attenuated oocysts protect goat kids from clinical coccidiosis. Veterinary Parasitology, v.199, p.8-17, 2014.

SHARMA, D.K.; AGRAWAL, N.; MANDAL, A.; NIGAM, P.; BHUSHAN, S. Coccidia and gastrintestinal nematode infections in semi-intensively managed Jakhrana goats of semi-arid region of India. Tropical and Subtropical Agroecosystems, v.11, p.135-139, 2009.

SILVA, T.P.; FACURY FILHO, E.J.; NUNES, A.B.V.; ALBUQUERQUE, F.H.M.A.R.; FERREIRA, P.M.; CARVALHO, A.V. Dinâmica da infecção natural por Eimeria spp. em cordeiros da raça Santa Inês criados em sistema semi-intensivo no Norte de Minas Gerais. Arquivo Brasileiro de Medicina Veterinária e Zootecnia, v.59, n.6, p.1468-1472, 2007.

STATISTICAL ANALYSIS SYSTEM. SAS: user's guide. Version 9.1 ed. Cary, USA: SAS Institute Inc, USA, 2003.

STEINFELDER, S.; LUCIUS, R.; GREIF, G.; POGONKA, T. Treatment of mice the anticcocidial drug Toltrazuril does not interfere with the development of a specific celluler intestinal immune response to Eimeria falciformis T. Parasitology Research, v.97, n.6, p.458-465, 2005.
UENO, H.; GONÇALVES, P.C. Manual para o diagnóstico das helmintoses de ruminantes. 4.ed. Tokyo: Japan Internatinal Cooperation Agency, 1998. 143p.

VELLOSO, A.L.; SAMPAIO, E.V.S.; PAREYN, F S.G.C. Ecorregiões propostas para o bioma Caatinga. Recife: Associação Plantas do Nordeste, 2002. 76 p.

VIEIRA, L.S.; BARROS, N.N.; CAVALCANTE, A.C.R.; XIMENES, L.J.F.; CARVALHO, R.B. A salinomicina para o controle da eimeriose de caprinos leiteiros nas fases de cria e recria. Ciência Rural, v.34, n.3, p.873-878, 2004.

Data de recebimento: 15/07/2014

Data de aprovação: 19/12/2014 